Supporting Information

\title{
Influence of Steric Hindrance on the Core Geometry and Sulfoxidation Chemistry of Carboxylate-Rich Diiron(II) Complexes
}

\author{
Erwin Reisner, Tanya C. Abikoff and Stephen J. Lippard*
}

Department of Chemistry, Massachusetts Institute of Technology, Cambridge, Massachusetts 02139 
X-Ray Diffraction Refinement Details for Complexes 2, 3, 5, 6, and 7-14. Crystal data, data collection parameters, and structure refinement details for 2,3 , and 5-14 are provided in Tables S1-S3. Two disordered $\mathrm{CH}_{2} \mathrm{Cl}_{2}$ molecules at $40 \%$ and $60 \%$ occupancy were refined in the asymmetric unit of $\mathbf{2}$ and one disordered $\mathrm{CH}_{2} \mathrm{Cl}_{2}$ molecule at $25 \%$ occupancy was included per molecule of 3 . In 2 and 3 , one or two carbon atoms of the THF molecule are disordered over two positions, respectively. In addition, one terminal terphenylcarboxylate ligand in $\mathbf{3}$ is disordered over two positions and refined with a 55/45 occupancy ratio. Two $\mathrm{CH}_{2} \mathrm{Cl}_{2}$ molecules per asymmetric unit are disordered over a total of four positions in $\mathbf{5}$. No solvent molecules were identified in the crystal lattice of $\mathbf{6}$. Complex $\mathbf{7}$ crystallizes with one molecule of dichloromethane and one molecule of diethyl ether per asymmetric unit. Complex 8 contains two solvent molecules of $\mathrm{CH}_{2} \mathrm{Cl}_{2}$, and the ethyl group of the tethered substrate is disordered over two positions with a occupancy $3 / 7$ ratio. Complex 9 crystallizes with one chlorobenzene molecule in full and one pentane molecule in $25 \%$ occupancy. There are two $\mathrm{CH}_{2} \mathrm{Cl}_{2}$ molecules; one of them at half occupancy and the other one disordered over two positions, and an additional half-occupied chlorobenzene solvent molecule per diiron molecule in 10. Complexes 11 and 12 crystallized with two disordered molecules of chlorobenzene refined at half and full occupancy, respectively. In addition, 12 contains half a molecule of pentane, which was refined only isotropically. Two methyl groups of one ${ }^{-} \mathrm{O}_{2} \mathrm{CAr}{ }^{\mathrm{Ph}, \mathrm{Xyl}}$ ligand in compound 13 are disordered in a $\sim 1: 1$ ratio and two disordered chlorobenzene molecules with $75 \%$ and $25 \%$ occupancy were located, as well as a pentane molecule at $25 \%$ occupancy. One $\mathrm{CH}_{2} \mathrm{Cl}_{2}$ molecule is disordered in 14 over two positions and one diethyl ether molecule is observed at $75 \%$ occupancy. 
Table S1. Crystal Data and Details of Data Collection for 2, 3, 5 and 6.

\begin{tabular}{|c|c|c|c|c|}
\hline & $2 \cdot 2 \mathrm{CH}_{2} \mathrm{Cl}_{2}$ & $3 \cdot 1 / 2 \mathrm{CH}_{2} \mathrm{Cl}_{2}$ & $5 \cdot 4 \mathrm{CH}_{2} \mathrm{Cl}_{2}$ & 6 \\
\hline chemical formula & $\mathrm{C}_{94} \mathrm{H}_{88} \mathrm{Cl}_{4} \mathrm{Fe}_{2} \mathrm{O}_{10}$ & $\mathrm{C}_{92.5} \mathrm{H}_{85} \mathrm{ClFe}_{2} \mathrm{O}_{10}$ & $\mathrm{C}_{98} \mathrm{H}_{86} \mathrm{Cl}_{8} \mathrm{Fe}_{2} \mathrm{~N}_{2} \mathrm{O}_{8}$ & $\mathrm{C}_{94} \mathrm{H}_{78} \mathrm{Fe}_{2} \mathrm{~N}_{2} \mathrm{O}_{8}$ \\
\hline fw $(\mathrm{g} / \mathrm{mol})$ & 1631.20 & 1503.80 & 1815.05 & 1475.32 \\
\hline space group & $\mathrm{P} 2{ }_{1} / \mathrm{c}$ & $\mathrm{P} 2{ }_{1} / \mathrm{n}$ & $\mathrm{P} 22_{1} / \mathrm{c}$ & $\mathrm{P} \overline{1}$ \\
\hline $\mathrm{a}(\AA)$ & $12.2604(12)$ & $12.9614(19)$ & $12.313(4)$ & 11.707(3) \\
\hline $\mathrm{b}(\AA)$ & $17.6170(17)$ & $23.089(3)$ & $17.466(6)$ & $14.289(4)$ \\
\hline$c(\AA)$ & $20.0356(19)$ & $13.1367(19)$ & $20.457(7)$ & $24.133(6)$ \\
\hline$\alpha(\operatorname{deg})$ & & & & $93.286(5)$ \\
\hline$\beta$ (deg) & $92.308(2)$ & $99.373(2)$ & $93.745(7)$ & $95.981(5)$ \\
\hline$\gamma(\mathrm{deg})$ & & & & $111.166(4)$ \\
\hline$V\left(\AA^{3}\right)$ & $4324.0(7)$ & $3878.9(9)$ & $4390(3)$ & $3724.6(16)$ \\
\hline Z & 2 & 2 & 2 & 2 \\
\hline$\rho_{\text {calc }}\left(\mathrm{g} / \mathrm{cm}^{3}\right)$ & 1.253 & 1.288 & 1.373 & 1.315 \\
\hline $\mathrm{T}(\mathrm{K})$ & 123 & 110 & 110 & 110 \\
\hline crystal description & colorless block & colorless block & pale yellow plate & yellow block \\
\hline crystal size (mm) & $0.20 \times 0.15 \times 0.10$ & $0.40 \times 0.10 \times 0.10$ & $0.40 \times 0.25 \times 0.06$ & $0.20 \times 0.12 \times 0.10$ \\
\hline sec/frame & 20 & 10 & 20 & 20 \\
\hline$\mu\left(\mathrm{Mo} \mathrm{K}_{\alpha}\right), \mathrm{mm}^{-1}$ & 0.516 & 0.469 & 0.633 & 0.452 \\
\hline$\Theta$ range, deg & $2.30-25.03$ & $2.05-25.68$ & $2.00-25.03$ & $2.18-25.03$ \\
\hline total no. of data & 57379 & 56306 & 56684 & 52378 \\
\hline no. of unique data & 7643 & 7358 & 7748 & 13125 \\
\hline no. of parameters & 593 & 736 & 592 & 963 \\
\hline completeness & $99.9 \%$ & $100 \%$ & $99.9 \%$ & $99.8 \%$ \\
\hline method of refinement & Patterson & direct & Patterson & direct \\
\hline $\mathrm{R} 1^{\mathrm{a}}$ & 0.0617 & 0.0594 & 0.0618 & 0.0626 \\
\hline$w R 2^{b}$ & 0.1741 & 0.1337 & 0.1313 & 0.1154 \\
\hline $\mathrm{GOF}^{\mathrm{c}}$ & 1.082 & 1.193 & 1.098 & 1.052 \\
\hline
\end{tabular}

${ }^{\mathrm{a}} \mathrm{R} 1=\Sigma|| F_{\mathrm{o}}|-| F_{\mathrm{c}}|| / \Sigma\left|F_{\mathrm{o}}\right|,{ }^{\mathrm{b}} w \mathrm{R}^{2}=\left\{\Sigma\left[w\left(F_{\mathrm{o}}{ }^{2}-F_{\mathrm{c}}{ }^{2}\right)^{2}\right] / \Sigma\left[w\left(F_{\mathrm{o}}{ }^{2}\right)^{2}\right]\right\}^{1 / 2},{ }^{\mathrm{c}} \mathrm{GOF}=\left\{\Sigma\left[w\left(F_{\mathrm{o}}^{2}-F_{\mathrm{c}}{ }^{2}\right)^{2}\right]\right.$ $/(\mathrm{n}-\mathrm{p})\}^{1 / 2}$, where $\mathrm{n}$ is the number of reflections and $\mathrm{p}$ is the total number of parameters refined. 
Table S2. Crystal Data and Details of Data Collection for Compounds 7-10.

\begin{tabular}{|c|c|c|c|c|}
\hline & $7 \cdot \mathrm{CH}_{2} \mathrm{Cl}_{2} \cdot \mathrm{Et}_{2} \mathrm{O}$ & $8 \cdot 2 \mathrm{CH}_{2} \mathrm{Cl}_{2}$ & 9. $\mathrm{C}_{6} \mathrm{H}_{5} \mathrm{Cl} \cdot 1 / 4 \mathrm{C}_{5} \mathrm{H}_{12}$ & $10 \cdot 1.5 \mathrm{CH}_{2} \mathrm{Cl}_{2} \cdot 0.5 \mathrm{C}_{6} \mathrm{H}_{5} \mathrm{Cl}$ \\
\hline chemical formula & $\mathrm{C}_{96} \mathrm{H}_{89} \mathrm{Cl}_{2} \mathrm{Fe}_{2} \mathrm{NO}_{9} \mathrm{~S}$ & $\mathrm{C}_{94} \mathrm{H}_{83} \mathrm{Cl}_{4} \mathrm{Fe}_{2} \mathrm{NO}_{8} \mathrm{~S}$ & $\mathrm{C}_{101.25} \mathrm{H}_{91} \mathrm{ClFe}_{2} \mathrm{NO}_{8} \mathrm{~S}$ & $\mathrm{C}_{100.5} \mathrm{H}_{84.5} \mathrm{Cl}_{3.5} \mathrm{Fe}_{2} \mathrm{NO}_{8} \mathrm{~S}$ \\
\hline $\mathrm{fw}(\mathrm{g} / \mathrm{mol})$ & 1615.34 & 1640.17 & 1628.98 & 1702.03 \\
\hline space group & $\mathrm{P} 2_{1} / \mathrm{c}$ & $\mathrm{P} 2_{1} / \mathrm{c}$ & $\mathrm{P} 22_{1}{ }_{2}$ & $\mathrm{P} 2_{1} / \mathrm{n}$ \\
\hline$a(\AA)$ & $12.9047(7)$ & $12.9053(10)$ & $25.1545(18)$ & $16.5043(11)$ \\
\hline $\mathrm{b}(\AA)$ & $27.1966(15)$ & $26.771(2)$ & $25.3801(17)$ & $26.2368(15)$ \\
\hline$c(\AA)$ & $23.0779(13)$ & $23.6564(19)$ & $13.7159(9)$ & $21.0392(12)$ \\
\hline \multicolumn{5}{|l|}{$\alpha(\mathrm{deg})$} \\
\hline$\beta(\mathrm{deg})$ & $92.6130(10)$ & $94.1390(10)$ & & $96.0190(10)$ \\
\hline \multicolumn{5}{|l|}{$\gamma(\mathrm{deg})$} \\
\hline$V\left(\AA^{3}\right)$ & $8091.1(8)$ & $8151.7(11)$ & $8756.6(10)$ & $9060.2(9)$ \\
\hline z & 4 & 4 & 4 & 4 \\
\hline$\rho_{\text {calc }}\left(\mathrm{g} / \mathrm{cm}^{3}\right)$ & 1.326 & 1.336 & 1.236 & 1.248 \\
\hline $\mathrm{T}(\mathrm{K})$ & 173 & 173 & 173 & 173 \\
\hline crystal description & Yellow needle & Yellow needle & Yellow block & Yellow needle \\
\hline crystal size $(\mathrm{mm})$ & $0.50 \times 0.10 \times 0.08$ & $0.40 \times 0.08 \times 0.08$ & $0.20 \times 0.20 \times 0.18$ & $0.20 \times 0.10 \times 0.08$ \\
\hline sec/frame & 10 & 20 & 10 & 20 \\
\hline$\mu\left(\mathrm{Mo} \mathrm{K}_{\alpha}\right), \mathrm{mm}^{-1}$ & 0.511 & 0.571 & 0.443 & 0.502 \\
\hline$\Theta$ range, deg & $2.32-25.68$ & $2.30-25.68$ & $2.28-28.30$ & $2.33-23.26$ \\
\hline total no. of data & 117721 & 114585 & 154746 & 108041 \\
\hline no. of unique data & 15354 & 15475 & 21770 & 13014 \\
\hline no. of parameters & 1008 & 1017 & 1059 & 1105 \\
\hline completeness to theta & $99.9 \%$ & $99.9 \%$ & $99.9 \%$ & $99.9 \%$ \\
\hline Flack parameter (esd) & & & $\begin{array}{c}0.0104 \\
(0.0121)\end{array}$ & \\
\hline method of refinement & direct & direct & direct & direct \\
\hline $\mathrm{R} 1^{\mathrm{a}}$ & 0.0557 & 0.0670 & 0.0597 & 0.0746 \\
\hline$w R 2^{b}$ & 0.1219 & 0.1656 & 0.1520 & 0.1979 \\
\hline $\mathrm{GOF}^{\mathrm{c}}$ & 1.049 & 1.093 & 1.094 & 1.035 \\
\hline
\end{tabular}


Table S3. Crystal Data and Details of Data Collection for Compounds 11-14.

\begin{tabular}{|c|c|c|c|c|}
\hline & $11 \cdot \mathrm{C}_{6} \mathrm{H}_{5} \mathrm{Cl}$ & $12 \cdot 2 \mathrm{C}_{6} \mathrm{H}_{5} \mathrm{Cl} \cdot 1 / 2 \mathrm{C}_{5} \mathrm{H}_{12}$ & $13 \cdot \mathrm{C}_{6} \mathrm{H}_{5} \mathrm{Cl} \cdot 1 / 4 \mathrm{C}_{5} \mathrm{H}_{12}$ & 14. $\mathrm{CH}_{2} \mathrm{Cl}_{2} \cdot 3 / 4 \mathrm{Et}_{2} \mathrm{O}$ \\
\hline chemical formula & $\mathrm{C}_{105} \mathrm{H}_{90} \mathrm{ClFe}{ }_{2} \mathrm{NO}_{8} \mathrm{~S}$ & $\mathrm{C}_{119.5} \mathrm{H}_{113} \mathrm{Cl}_{2} \mathrm{Fe}_{2} \mathrm{NO}_{8} \mathrm{~S}$ & $\mathrm{C}_{106.25} \mathrm{H}_{93} \mathrm{ClFe}_{2} \mathrm{NO}_{8} \mathrm{~S}$ & $\mathrm{C}_{96} \mathrm{H}_{88.5} \mathrm{Cl}_{2} \mathrm{Fe}_{2} \mathrm{NO}_{9.75}$ \\
\hline $\mathrm{fw}(\mathrm{g} / \mathrm{mol})$ & 1672.99 & 1905.77 & 1691.03 & 1594.78 \\
\hline space group & $\mathrm{P} 2{ }_{1} 2{ }_{1} 2$ & $\mathrm{P} \overline{1}$ & $\mathrm{P} 2_{1} / \mathrm{c}$ & $\mathrm{P} 2{ }_{1} / \mathrm{c}$ \\
\hline$a(\AA)$ & $24.7949(18)$ & $12.8583(8)$ & $19.538(19)$ & $12.9375(11)$ \\
\hline $\mathrm{b}(\AA)$ & $26.2886(19)$ & $15.4052(10)$ & $14.946(15)$ & 26.992(2) \\
\hline$c(\AA \AA)$ & $13.7704(10)$ & $27.3385(18)$ & $35.12(3)$ & $23.441(2)$ \\
\hline$\alpha$ (deg) & & $88.872(1)$ & & \\
\hline$\beta(\mathrm{deg})$ & & $78.052(1)$ & $105.542(18)$ & $93.893(2)$ \\
\hline$\gamma(\mathrm{deg})$ & & $71.666(1)$ & & \\
\hline$V\left(\AA^{3}\right)$ & $8975.9(11)$ & $5023.1(6)$ & $9880(17)$ & $8167(1)$ \\
\hline Z & 4 & 2 & 4 & 4 \\
\hline$\rho_{\text {calc }}\left(\mathrm{g} / \mathrm{cm}^{3}\right)$ & 1.238 & 1.260 & 1.137 & 1.297 \\
\hline $\mathrm{T}(\mathrm{K})$ & 173 & 173 & 110 & 173 \\
\hline crystal description & Yellow block & Yellow block & Yellow block & Yellow needle \\
\hline crystal size $(\mathrm{mm})$ & $0.30 \times 0.10 \times 0.08$ & $0.30 \times 0.20 \times 0.20$ & $0.30 \times 0.20 \times 0.05$ & $0.40 \times 0.04 \times 0.04$ \\
\hline sec/frame & 10 & 10 & 20 & 20 \\
\hline$\mu\left(\mathrm{Mo} \mathrm{K}_{\alpha}\right), \mathrm{mm}^{-1}$ & 0.434 & 0.422 & 0.395 & 0.482 \\
\hline$\Theta$ range, deg & $2.29-24.99$ & $2.51-26.37$ & $2.16-25.03$ & $2.30-28.33$ \\
\hline total no. of data & 125346 & 77782 & 105968 & 142456 \\
\hline no. of unique data & 15796 & 20441 & 17453 & 20259 \\
\hline no. of parameters & 1171 & 1336 & 1219 & 1036 \\
\hline completeness to theta & $99.8 \%$ & $99.5 \%$ & $99.9 \%$ & $99.5 \%$ \\
\hline $\begin{array}{l}\text { Flack parameter (esd) } \\
\text { method of refinement }\end{array}$ & $\begin{array}{c}0.0165 \\
(0.0127) \\
\text { direct }\end{array}$ & direct & direct & direct \\
\hline $\mathrm{R} 1^{\mathrm{a}}$ & 0.0538 & 0.0585 & 0.0858 & 0.0676 \\
\hline$w R 2^{b}$ & 0.1256 & 0.1535 & 0.2485 & 0.1519 \\
\hline $\mathrm{GOF}^{\mathrm{c}}$ & 1.054 & 1.085 & 1.089 & 1.071 \\
\hline
\end{tabular}

${ }^{\mathrm{a}} \mathrm{R} 1=\Sigma|| F_{\mathrm{o}}|-| F_{\mathrm{c}}|| / \Sigma\left|F_{\mathrm{o}}\right|,{ }^{\mathrm{b}} w \mathrm{R}^{2}=\left\{\Sigma\left[w\left(F_{\mathrm{o}}{ }^{2}-F_{\mathrm{c}}{ }^{2}\right)^{2}\right] / \Sigma\left[w\left(F_{\mathrm{o}}{ }^{2}\right)^{2}\right]\right\}^{1 / 2},{ }^{\mathrm{c}} \mathrm{GOF}=\left\{\Sigma\left[w\left(F_{\mathrm{o}}^{2}-F_{\mathrm{c}}{ }^{2}\right)^{2}\right]\right.$ $/(n-p)\}^{1 / 2}$, where $n$ is the number of reflections and $p$ is the total number of parameters refined. 


\section{Figure Captions.}

Figure S1. Top: ORTEP diagram and atom-labeling scheme for $\left[\mathrm{Fe}_{2}\left(\mu-\mathrm{O}_{2} \mathrm{CAr}{ }^{\mathrm{Tol}}\right)_{2^{-}}\right.$ $\left.\left(\mathrm{O}_{2} \mathrm{CAr}{ }^{\mathrm{Ph}, \mathrm{Xyl}}\right)_{2}(\mathrm{THF})_{2}\right](2)$ depicting positional disorder of one carbon atom in each THF molecule. Hydrogen atoms and the aromatic rings of the carboxylate ligands are omitted for clarity. Bottom: ORTEP diagram of complex 2 showing 50\% probability thermal ellipsoids for all non-hydrogen atoms.

Figure S2. Top: ORTEP diagram and atom-labeling scheme for $\left[\mathrm{Fe}_{2}\left(\mu-\mathrm{O}_{2} \mathrm{CAr} r^{\mathrm{Ph}, \mathrm{Xyl}}\right)_{2^{-}}\right.$ $\left.\left(\mathrm{O}_{2} \mathrm{CAr}{ }^{\mathrm{Ph}, \mathrm{Xyl}}\right)_{2}(\mathrm{THF})_{2}\right](3)$ depicting positional disorder of two carbon atoms in each THF molecule. Hydrogen atoms and the aromatic rings of the carboxylate ligands omitted for clarity. Bottom: ORTEP diagram of complex 3 showing $50 \%$ probability thermal ellipsoids for all non-hydrogen atoms.

Figure S3. Top: ORTEP diagram and atom-labeling scheme for $\left[\mathrm{Fe}_{2}(\mu\right.$ $\left.\left.\mathrm{O}_{2} \mathrm{CAr}^{\mathrm{Tol}}\right)_{2}\left(\mathrm{O}_{2} \mathrm{CAr}{ }^{\mathrm{Ph}, \mathrm{Xyl}}\right)_{2}(\text { pyridine })_{2}\right]$ (5). Hydrogen atoms and the aromatic rings of the carboxylate ligands omitted for clarity. Bottom: ORTEP diagram of complex $\mathbf{5}$ showing $50 \%$ probability thermal ellipsoids for all non-hydrogen atoms.

Figure S4. Top: ORTEP diagram and atom-labeling scheme for $\left[\mathrm{Fe}_{2}(\mu-\right.$ $\left.\left.\mathrm{O}_{2} \mathrm{CAr}^{\mathrm{Ph}, \mathrm{Xyl}}\right)_{2}\left(\mathrm{O}_{2} \mathrm{CAr}{ }^{\mathrm{Ph}, \mathrm{Xyl}}\right)_{2}(\text { pyridine })_{2}\right](6)$. Hydrogen atoms and the aromatic rings of the carboxylate ligands omitted for clarity. Bottom: ORTEP diagram of complex 6 showing $50 \%$ probability thermal ellipsoids for all non-hydrogen atoms. 
Figure S5. Top: ORTEP diagram and atom-labeling scheme for $\left[\mathrm{Fe}_{2}(\mu\right.$ $\left.\mathrm{O}_{2} \mathrm{CAr}^{\mathrm{Tol}}\right)_{3}\left(\mathrm{O}_{2} \mathrm{CAr}^{\mathrm{Tol}}\right)$ (picSMe)] (7). Hydrogen atoms and the aromatic rings of the carboxylate ligands omitted for clarity. Bottom: ORTEP diagram of complex 7 showing $50 \%$ probability thermal ellipsoids for all non-hydrogen atoms.

Figure S6. Top: ORTEP diagram and atom-labeling scheme for $\left[\mathrm{Fe}_{2}(\mu-\right.$ $\left.\mathrm{O}_{2} \mathrm{CAr}^{\mathrm{Tol}}\right)_{3}\left(\mathrm{O}_{2} \mathrm{CAr}{ }^{\mathrm{Tol}}\right)$ (picSEt)] (8). Hydrogen atoms and the aromatic rings of the carboxylate ligands omitted for clarity. One part of the disordered ethyl group in $\mathbf{8}$ is drawn as a dashed line. Bottom: ORTEP diagram of complex 8 showing $50 \%$ probability thermal ellipsoids for all non-hydrogen atoms.

Figure S7. Top: ORTEP diagram and atom-labeling scheme for $\left[\mathrm{Fe}_{2}(\mu\right.$ $\left.\left.\mathrm{O}_{2} \mathrm{CAr}^{\mathrm{Tol}}\right)_{3}\left(\mathrm{O}_{2} \mathrm{CAr}^{\mathrm{Tol}}\right)\left(\mathrm{picS}^{\mathrm{t}} \mathrm{Bu}\right)\right]$ (9). Hydrogen atoms and the aromatic rings of the carboxylate ligands omitted for clarity. Bottom: ORTEP diagram of complex 9 showing $50 \%$ probability thermal ellipsoids for all non-hydrogen atoms.

Figure S8. Top: ORTEP diagram and atom-labeling scheme for $\left[\mathrm{Fe}_{2}(\mu-\right.$ $\left.\left.\mathrm{O}_{2} \mathrm{CAr}^{\mathrm{Tol}}\right)_{3}\left(\mathrm{O}_{2} \mathrm{CAr}^{\mathrm{Tol}}\right)(\mathrm{picSPh})\right](\mathbf{1 0})$. Hydrogen atoms and the aromatic rings of the carboxylate ligands omitted for clarity. Bottom: ORTEP diagram of complex 10 showing $50 \%$ probability thermal ellipsoids for all non-hydrogen atoms. 
Figure S9. Top: ORTEP diagram and atom-labeling scheme for $\left[\mathrm{Fe}_{2}(\mu\right.$ $\left.\mathrm{O}_{2} \mathrm{CAr}^{\mathrm{Tol}}\right)_{3}\left(\mathrm{O}_{2} \mathrm{CAr}^{\mathrm{Tol}}\right)\left(\right.$ picSPh$\left.\left.\left(\mathrm{Me}_{3}\right)\right)\right]$ (11). Hydrogen atoms and the aromatic rings of the carboxylate ligands omitted for clarity. Bottom: ORTEP diagram of complex 11 showing $50 \%$ probability thermal ellipsoids for all non-hydrogen atoms.

Figure S10. Top: ORTEP diagram and atom-labeling scheme for $\left[\mathrm{Fe}_{2}(\mu-\right.$ $\left.\mathrm{O}_{2} \mathrm{CAr}^{\mathrm{Tol}}\right)_{3}\left(\mathrm{O}_{2} \mathrm{CAr}^{\mathrm{Tol}}\right)\left(\right.$ picSPh$\left.\left.\left({ }^{\mathrm{i}} \mathrm{Pr}_{3}\right)\right)\right]$ (12). Hydrogen atoms and the aromatic rings of the carboxylate ligands omitted for clarity. Bottom: ORTEP diagram of complex 12 showing $50 \%$ probability thermal ellipsoids for all non-hydrogen atoms.

Figure S11. Top: ORTEP diagram and atom-labeling scheme for $\left[\mathrm{Fe}_{2}(\mu-\right.$ $\left.\left.\mathrm{O}_{2} \mathrm{CAr}^{\mathrm{Ph}, \mathrm{Xyl}}\right)_{3}\left(\mathrm{O}_{2} \mathrm{CAr}{ }^{\mathrm{Ph}, \mathrm{Xyl}}\right)\left(\operatorname{picSPh}\left(\mathrm{Me}_{3}\right)\right)\right](13)$. Hydrogen atoms and the aromatic rings of the carboxylate ligands omitted for clarity. Bottom: ORTEP diagram of complex 13 showing $50 \%$ probability thermal ellipsoids for all non-hydrogen atoms.

Figure S12. Top: ORTEP diagram and atom-labeling scheme for $\left[\mathrm{Fe}_{2}(\mu-\right.$ $\left.\mathrm{O}_{2} \mathrm{CAr}^{\mathrm{Tol}}\right)_{3}\left(\mathrm{O}_{2} \mathrm{CAr}^{\mathrm{Tol}}\right)($ picOEt)] (14). Hydrogen atoms and the aromatic rings of the carboxylate ligands omitted for clarity. Bottom: ORTEP diagram of complex 14 showing $50 \%$ probability thermal ellipsoids for all non-hydrogen atoms. 

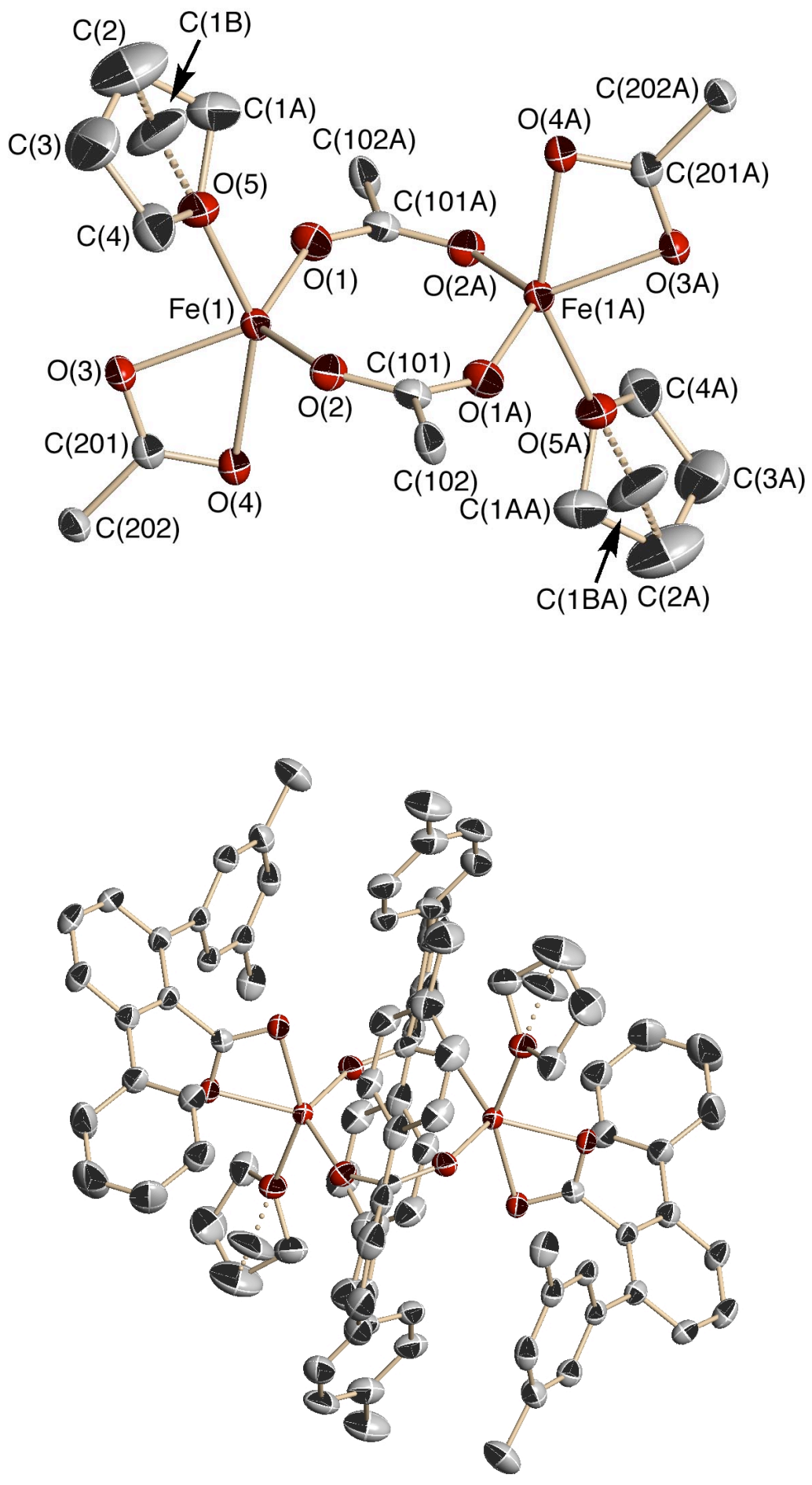

Figure S1. 

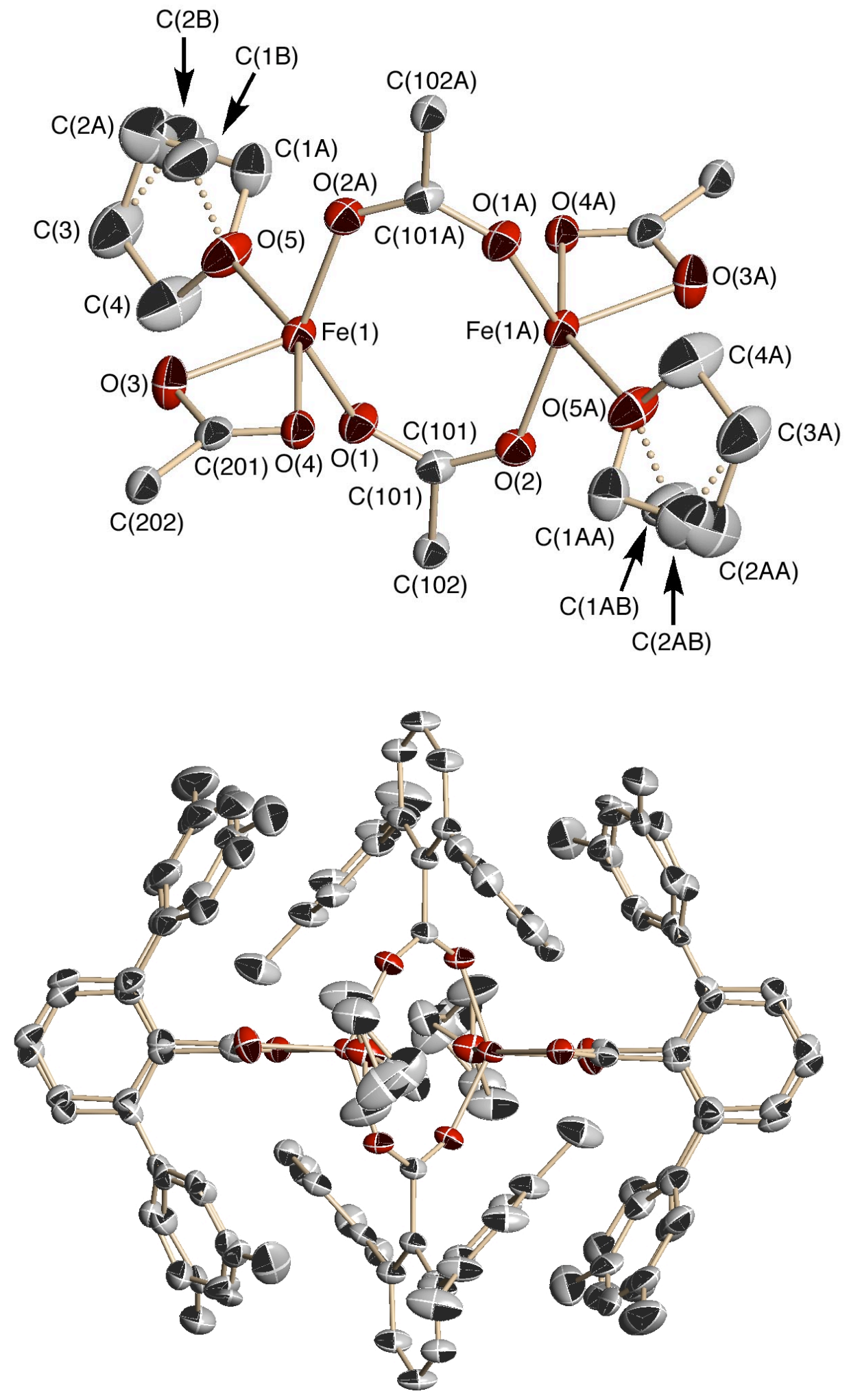

Figure S2. 

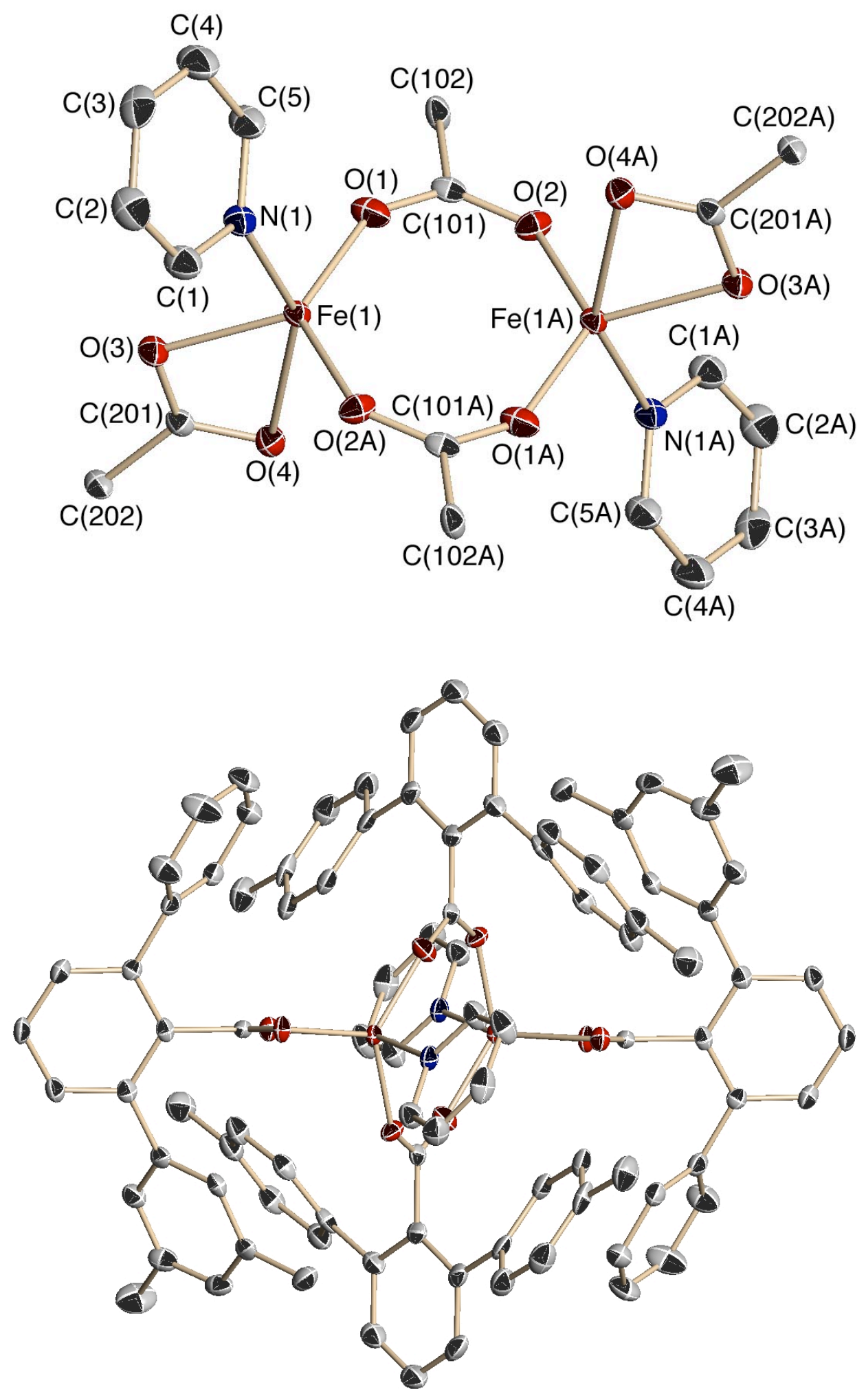

Figure S3. 

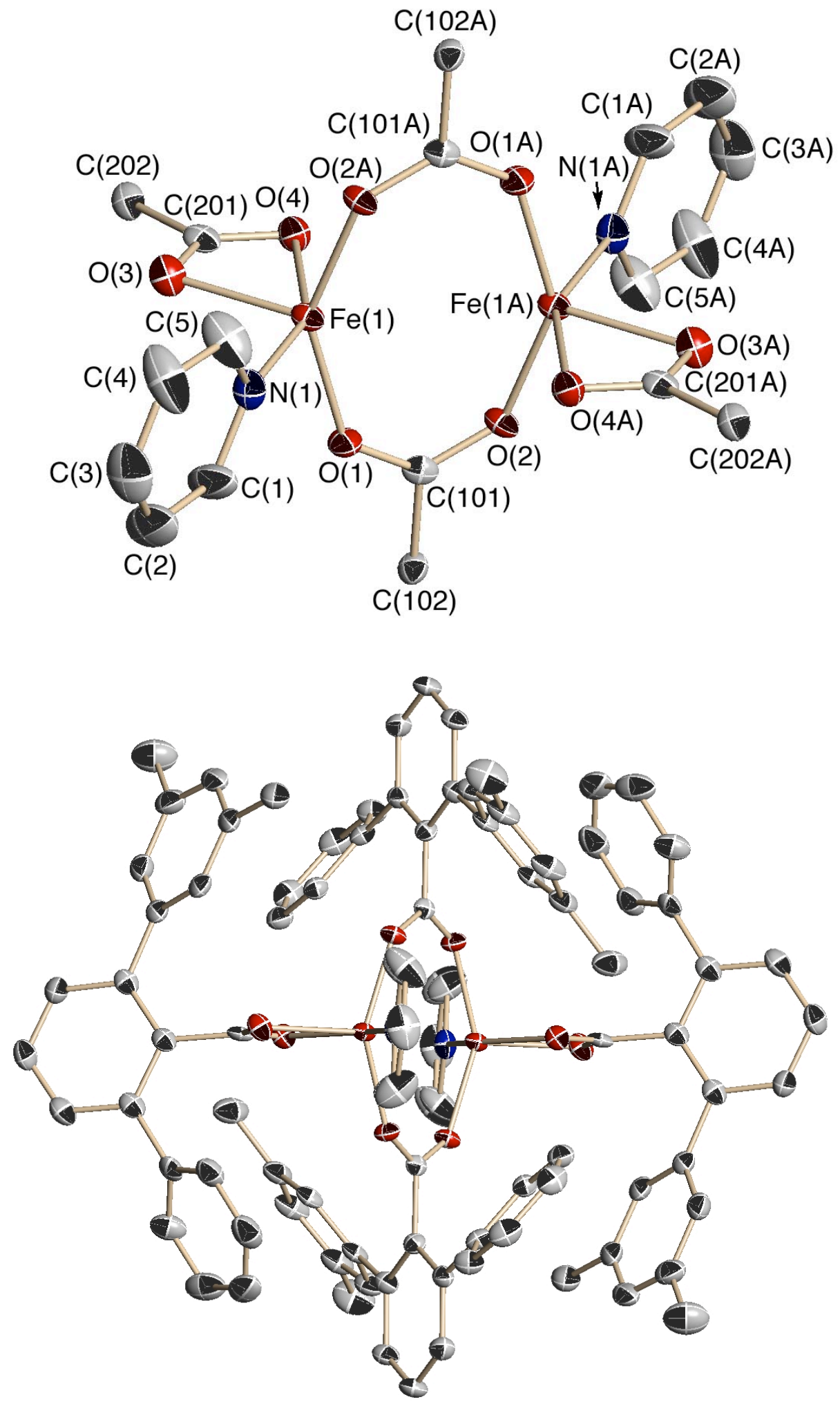

Figure S4. 

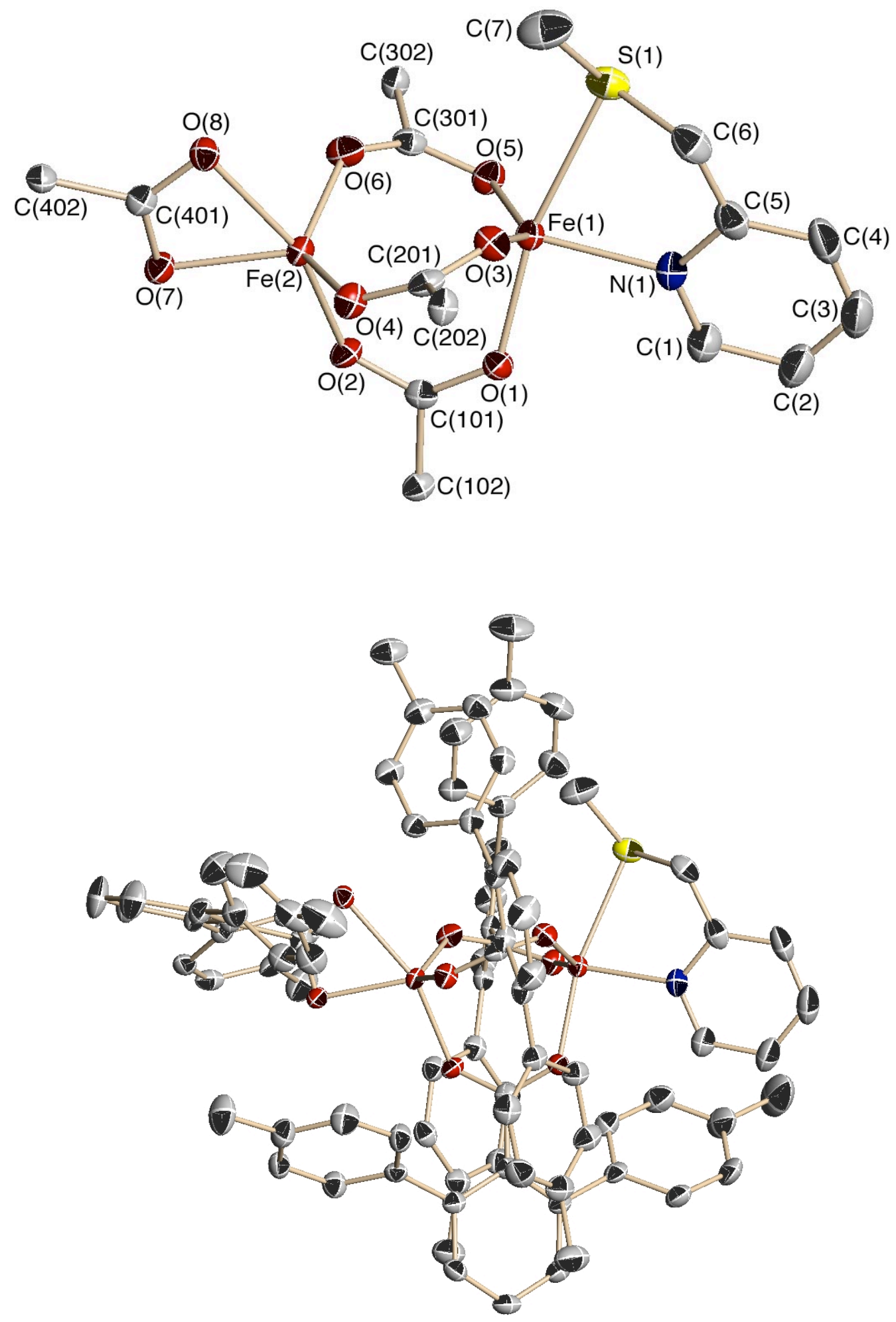

Figure S5. 

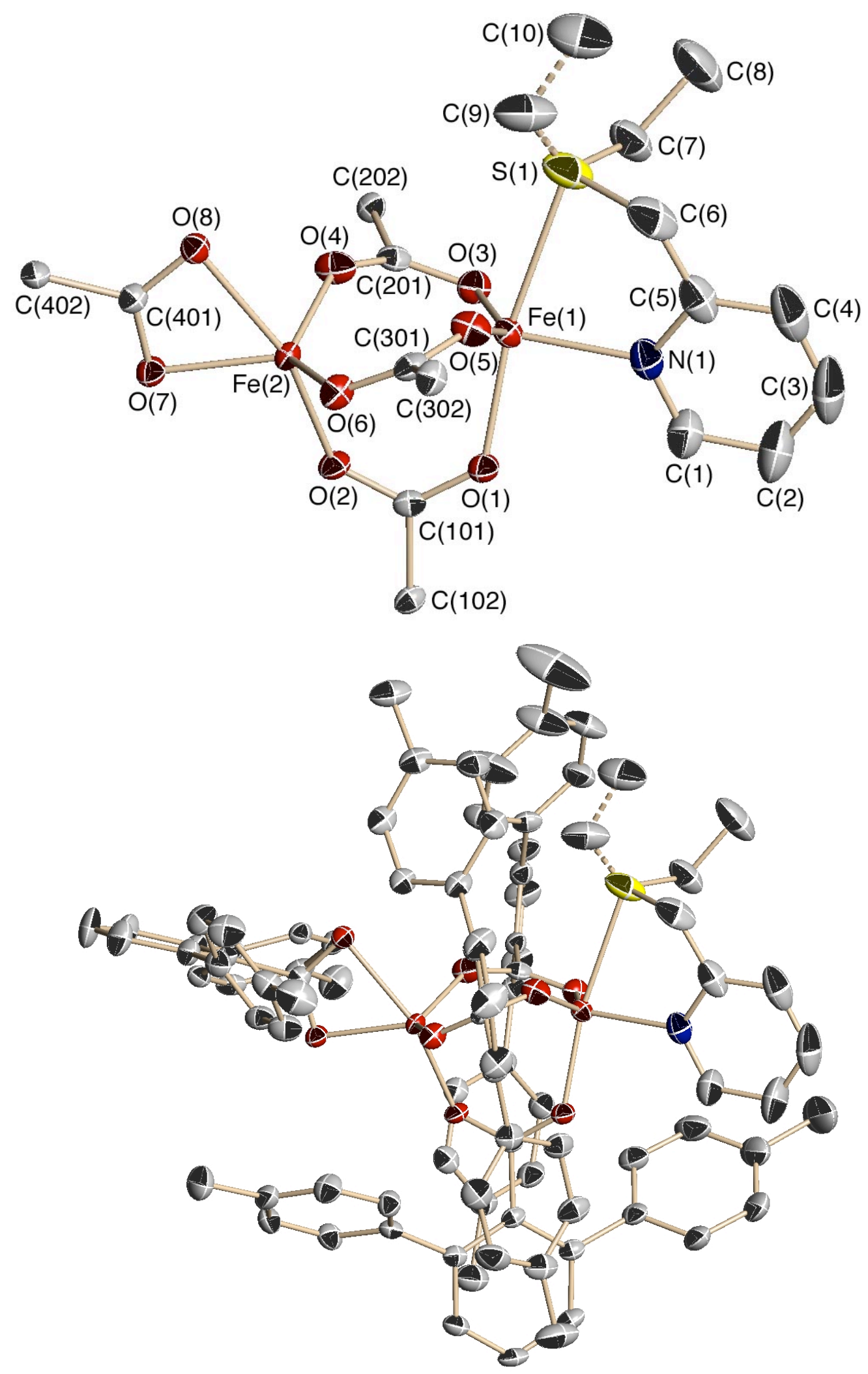

Figure S6. 

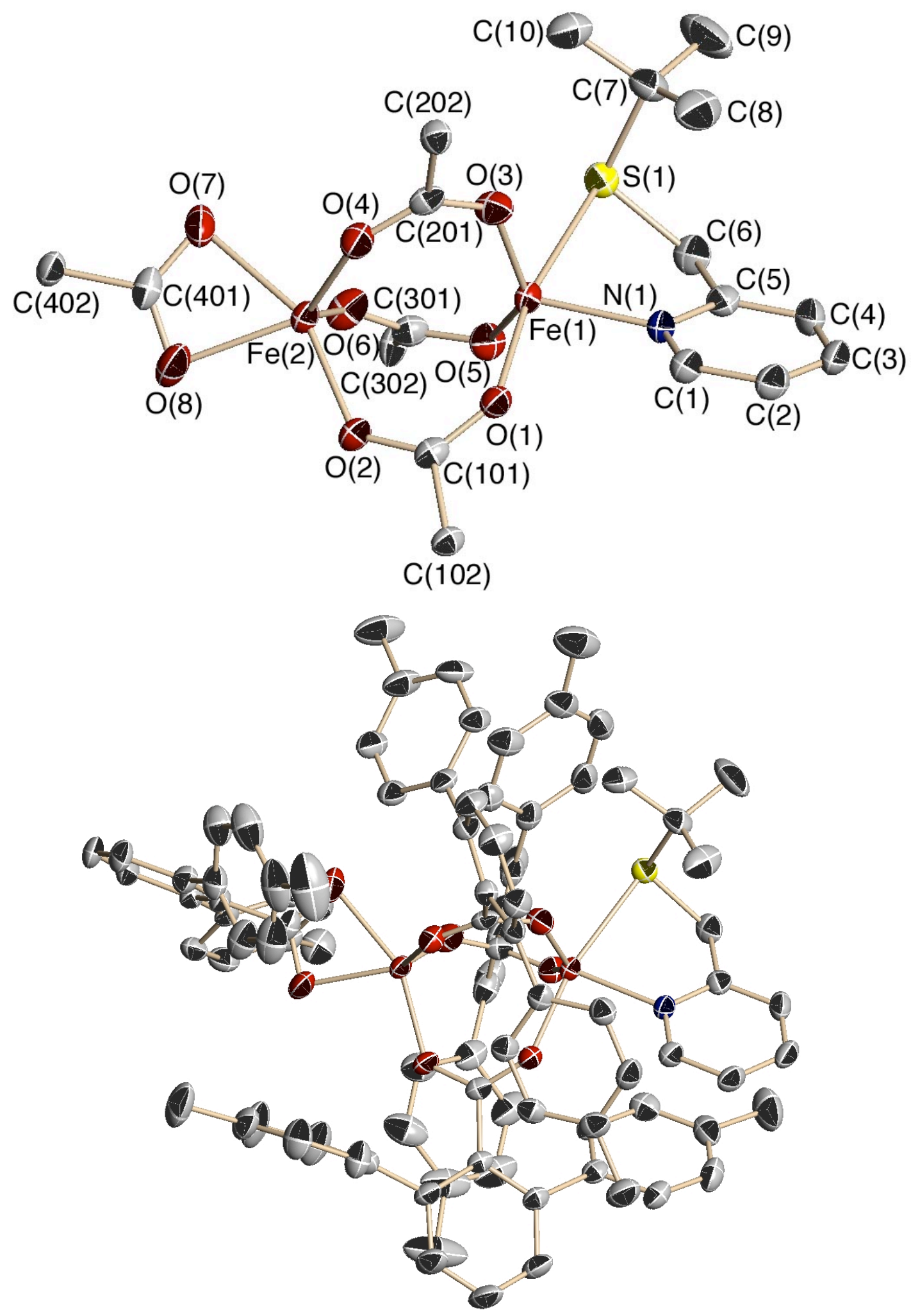

Figure S7. 

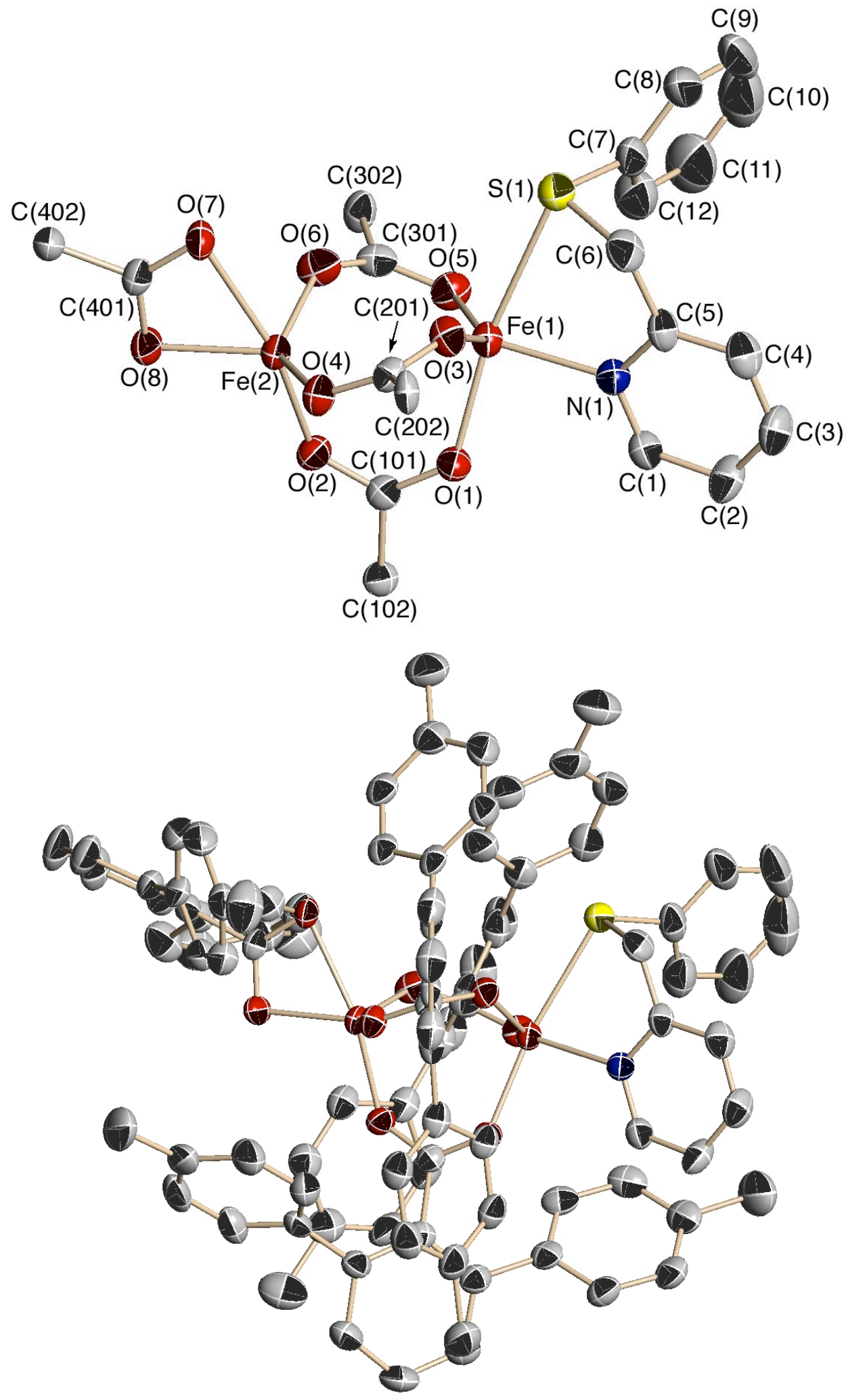

Figure S8. 

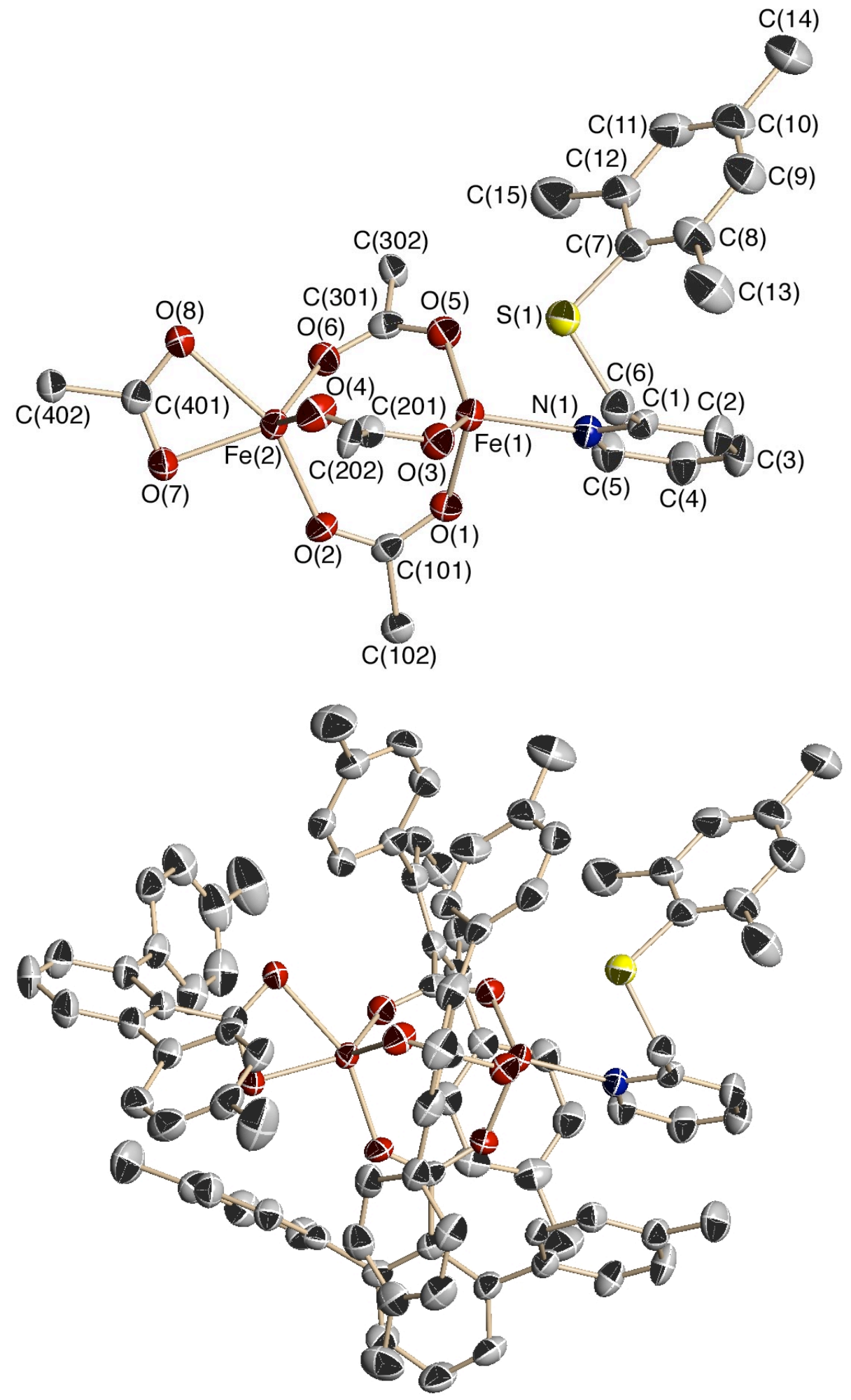

Figure S9. 

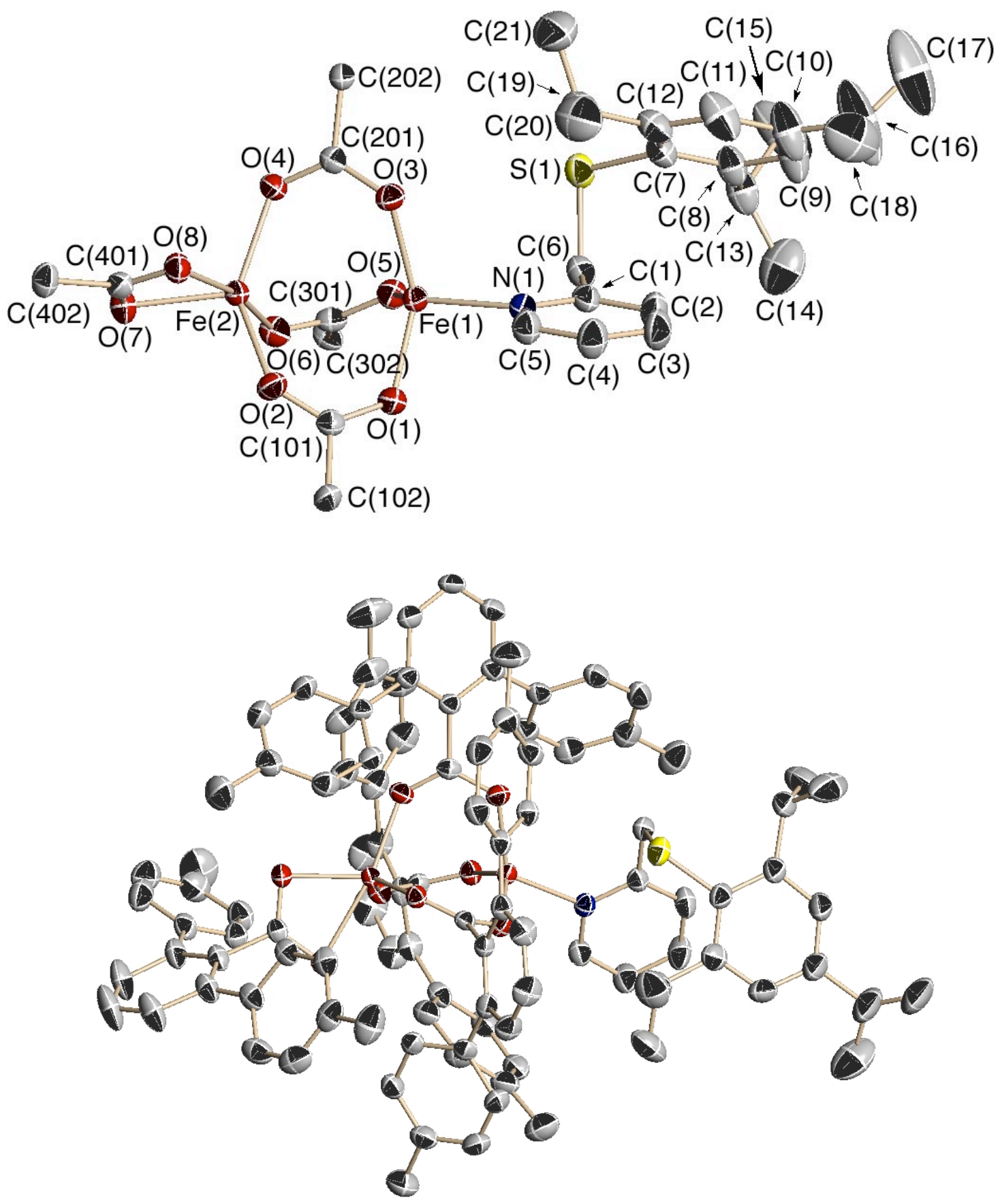

Figure S10. 

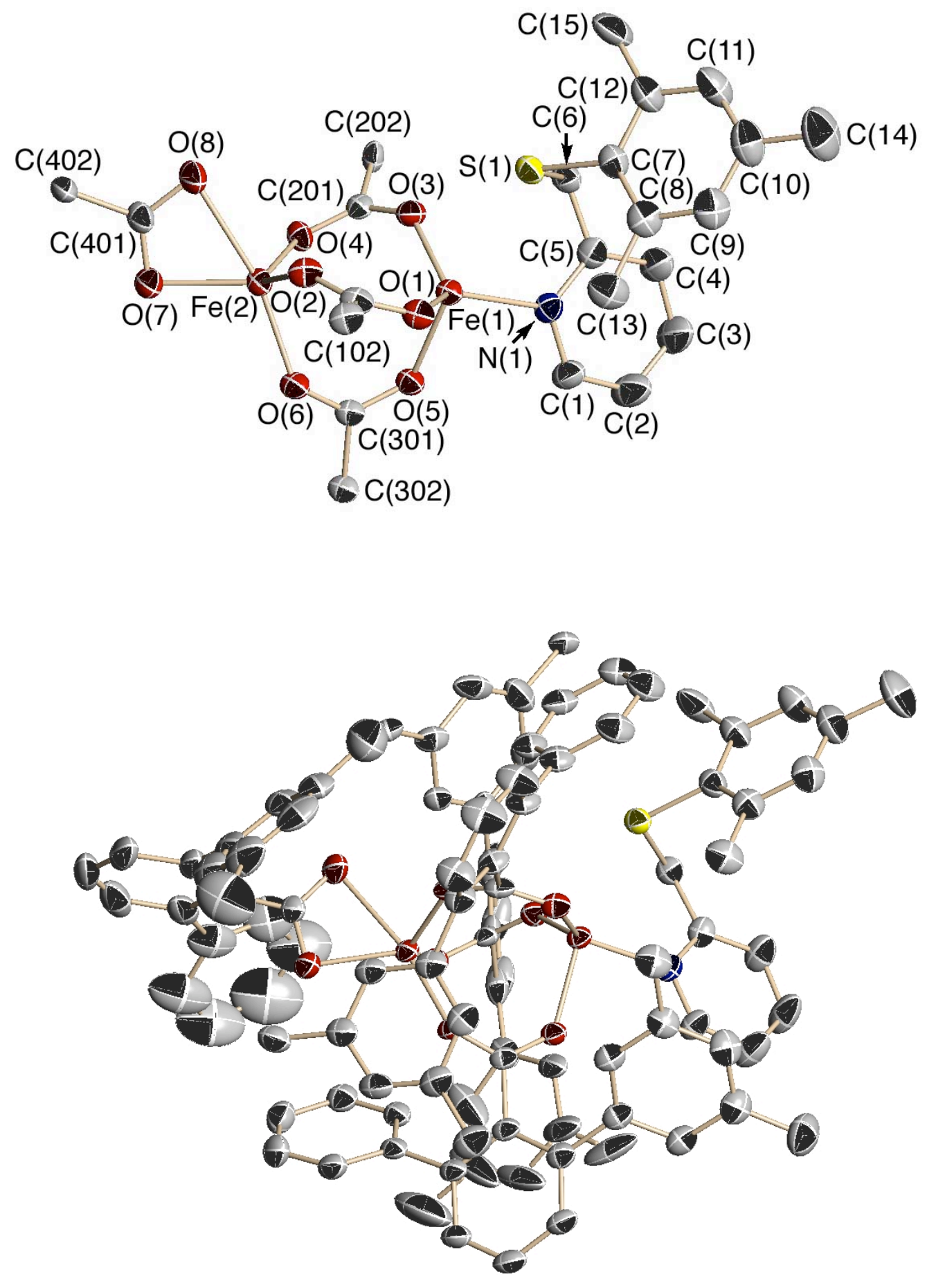

Figure S11. 

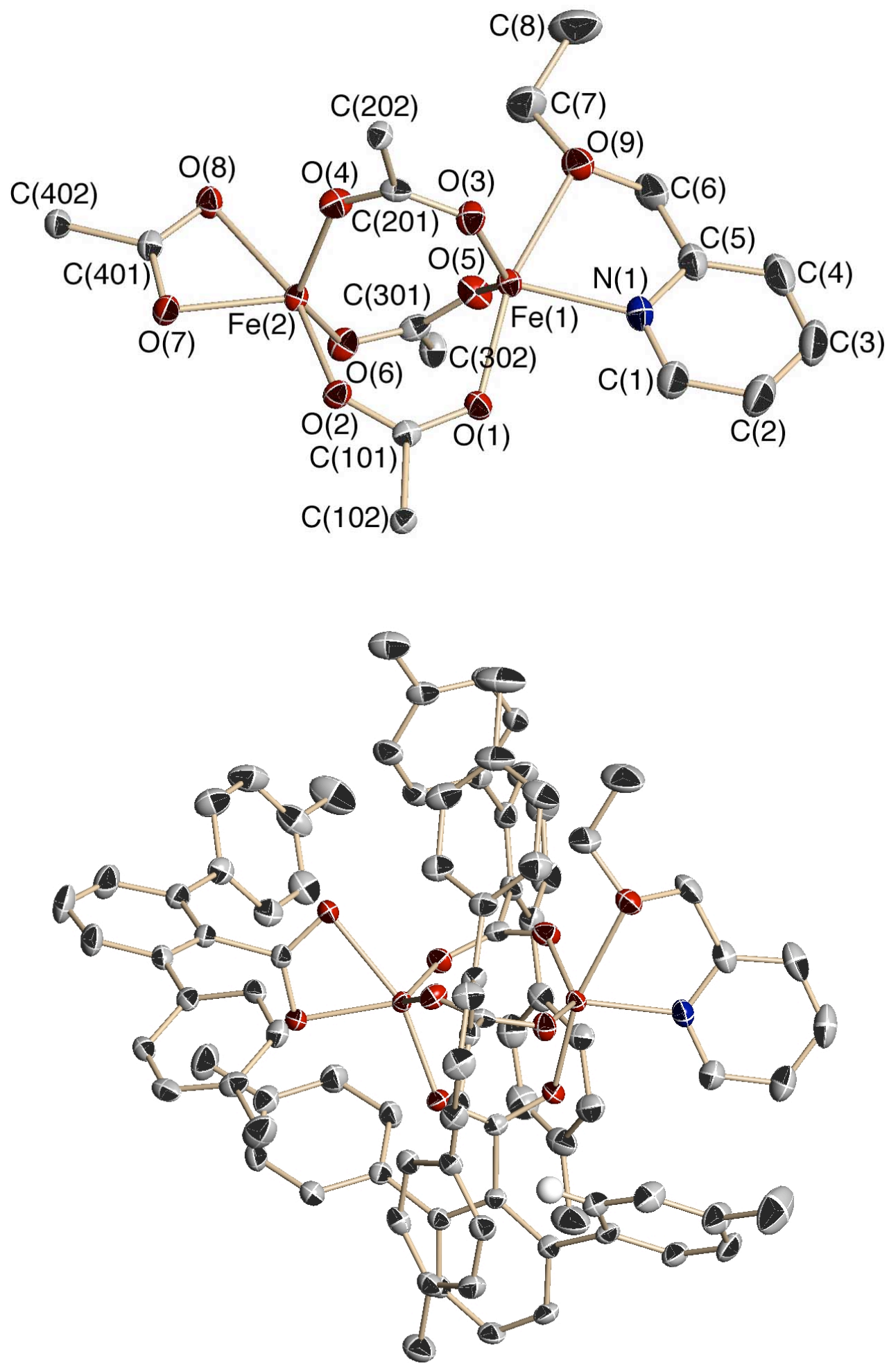

Figure S12. 